\title{
Dynamic response of inclined pipe conveying fluid under thermal effect
}

\author{
Jabbar. H. Mohmmed*, Mauwafak A. Tawfik, Qasim Abbas Atiyah \\ Mechanical Engineering Department, University of Technology-Iraq, Industrial St., Al- \\ Karrada District, Baghdad Province, Iraq
}

\begin{abstract}
This study treats with the transverse vibration of polypropylene random-copolymer (PP-R) pipes caused by fluid flow inside them assuming pinned connections at both ends. The effect of inclination angle, aspect ratio (the ratio of length to outside diameter) and temperature variation on dynamic response of inclined pipe containing flowing fluid with different velocity was investigated. The Euler-Bernoulli formula for beam theory was adopted to model the inclined pipe. An analytical model has been prepared to calculate the dynamic response of the pipe, taking into account pipe weight, thermal effect, inclination angle, aspect ratio, and fluid flow velocity, using the integral transform techniques (ITT) by utilizing a combining of Laplace and Fourier transforms and their inverses. The results demonstrate that the prepared analytic model is a powerful tool to obtain the dynamic characteristic of pipe conveying fluid. Moreover, the results showed that the dynamic deflection was strongly affected by the change in the values of inclination angle, pipe temperature, aspect ratio, and fluid velocity, where there was a significant increase in pipe deflection with increasing temperature and aspect ratio. and fluid velocity, while a decrease in the deflection value is observed with an increase in the angle of inclination in the range of $0-90^{\circ}$. The findings proved that the thermal effect becomes more important than the fluid velocity at high aspect ratios. The same case applies to the angle of inclination, as its effect on the pipe deflection increases at high aspect ratios. These results were limited to the fundamental (first) mode and can be useful for engineering component design. The main contributions of this work are to find the combined effect of inclination angle, thermal loading, and aspect ratio on the deflection of the pipe, in addition to preparing an analytical model to calculate this deflection.
\end{abstract}

Revised: August 25, 2021

Accepted: September 7, 2021

Corresponding Author:

Jabbar. H. Mohmmed

10493@uotechnology.edu.iq

Copyright: The Author(s). This is an open access article distributed under the terms of the Creative Commons Attribution License (CC BY 4.0), which permits unrestricted distribution provided the original author and source are cited.

\section{Publisher:}

Chaoyang University of

Technology

ISSN: $1727-2394$ (Print)

ISSN: 1727-7841 (Online)

Keywords: Flow velocity, Dynamic response, Temperature variation, Finite Fourier sine transforms, Laplace transforms, inclined pipe conveying fluid.

\section{INTRODUCTION}

The pipe containing flowing fluid considers as a fundamental dynamical problem in the fluid-structure interactions (FSI) field (Païdoussis, 2014; Kutin and Bajsić, 2014). This topic, as a kind of important engineering structure, is widely existing in many practical applications. Sometimes, their function is just for convey liquids or gas, as in oil and gas pipelines, pumps suction and discharge lines, and urban water supply. In other applications, they form primary structural parts as in power plants, chemical industries, hydraulic systems, liquid-fuel rocket piping components, refrigerators, air-conditioners, heat exchangers, nuclear power, marine equipment applications, aeronautic and space field and so on (Jweeg and Ntayeesh, 2016; Jiang et al., 2020). The problem of pipelines containing flowing fluid belongs to the wider group of dynamical problems including axially moving continua like traveling strings bands, belts, magnetic tapes and chain saws (Paidousiss, 2008).

In pipe-fluid system, a pressure can be imposed on the wall of the pipe by the fluid flowing inside the pipe that deforms the pipe, leads to fluid induced vibration. Also, 
thermal induced vibration can be produced as result of nonuniform heat transfer due to difference in convective heat transfer over the surface of the pipe. Depending on the system conditions these forces may induce moderate or excessive structural vibrations, leading to structural instability. Therefore, analysis static and dynamic deflection of pipe element due to fluid and thermal loading is necessary for safe design and operation (Marakala et al., 2009; Lu et al., 2020). Hence, it is important research problem in science domain span.

Several scholars have investigated thermal and fluid induced vibration. Most of studies was performed to evaluate the relation between speed of fluid flow, pressure of the fluid and its effect on the vibration behaviour. The first attempt to evaluate the models of pipe conveying fluid was by Ashley and Haviland (1950) who analysis the vibration noticed in the Trans-Arabian Pipeline. However, their formula of the problem was erroneous, as revealed by Feodos'yev (1951), who present the first correct governing equation for describe the dynamic behavior of a simply supported pipe conveying fluid. Similar problem was investigated independently by Housner (1952) using a different approach. Feodos'yev and Housner provide the first right equation of fluid-structure coupling vibration of pipe containing flowing fluid. Their investigation concentrates on the dynamic behavior of simply supported pipe line containing flowing oil. They show that at adequately high fluid speeds the pipe can buckle, mainly similar to a column under axial compressive forces. Later, numerous articles on both the plain pipe and its variants were published to analysis the linear and nonlinear dynamics of pipes containing flowing fluid under various boundaries and conditions (Huang et al., 2020; Askarian et al., 2020; Cao et al., 2018; Li et al., 2018; Ameen et al., 2019; Jweeg and Mohammad, 2010; Liang et al., 2020). Zhai et al. (2013) studied the dynamic response of curved pipe continuing flowing fluid under random excitation by using finite element model. The dynamic behaviour of a straight, lateral moving supported pipe conveying fluid was analysed by $\mathrm{Li}$ et al. (2018). Then, the dynamic response was further studied for composite pipe and cantilevered pipe conveying fluid by Oke and Khulief (2020); Wang et al. (2018) respectively.

The aforementioned studies on the dynamic response of pipes conveying fluid were mostly concerned with the horizontal, vertical, and curved pipes responses. In practice, however, with the rapid development in modern technologies, there is an increasing trend towards inclined pipes, which have different diameters and are subject to fluctuating environmental conditions such as temperature changes. Thus, it is of importance to explore dynamic response of pipes conveying fluid by considering the effects of inclination angle, aspect ratio (ratio of length to outer diameter), and thermal loading.
On the other hand, because of the complexity of dynamic behaviour of pipe conveying fluid problem, unlike discrete systems (Thomson, 1988; Ali et al., 2021), it was noted that most of the previous studies were done through the use of numerical or approximate techniques like differential quadrature method (DQM) (Nejadi et al., 2021), Galerkin (ElNajjar and Daneshmand, 2020), transfer matrix (Tawfik et al. 2009), finite elements (Amini et al., 2020; Alshalal et al., 2021) and so on. There is little work was published to solve these problems analytically. The classical way to obtain analytical solution is clearly still very essential because it employs as a precise benchmark for approximate and numerical solutions.

The current work aimed at preparing an analytic solution using mixing of finite Fourier sine and Laplace transforms of the governing equation of simply supported pipe conveying fluid modelling as Euler-Bernoulli beam. The response of structure due to combined effect of inclination angle, ratio of length to diameter (aspect ratio) and thermal loading with different fluid velocities was analysed. To the authors' knowledge, there are no work has been reported on dynamic response of simply supported pipe conveying fluid subjected to the combination effect of these parameters, which makes the current research on this subject necessary.

The present study is organized as follows. Firstly, the dynamic response problem for the inclined pipe conveying fluid was introduced in Section 1. Secondly, in Section 2, the governing equation of inclined pipe conveying fluid under thermal loads was presented, and the discrete dynamic model was built, and then the hybrid finite Fourier sine-Laplace transforms was employed for obtained the analytic representation formula of dynamic response. The effects of fluid velocity, inclination angle, aspect ratio, and thermal loading on the dynamic response of pipe were investigated and discussed in Section 3. Finally, in Section 4 , some conclusions were drawn.

\section{MATERIALS AND METHODS}

\subsection{The Governing Equation of Motion}

The considered system is comprised of a uniform, thin, simple supported pipe of total length $\mathrm{L}$, inner diameter $\mathrm{D}_{\mathrm{i}}$, outer diameter $\mathrm{D}_{0}$, subjected to thermal loading $F_{n}$, and containing incompressible flowing fluid with constant velocity $u$, as seen in Fig. 1. The pipe is elastic with constant bending stiffness EI, constant mass per unit length $\mathrm{M}$, and constant cross sectional area $\mathrm{A}_{\mathrm{p}}$. The fluid has constant mass per unit length $\mathrm{m}$, constant cross-sectional flow area $\mathrm{A}$, and its profile is uniform. Damping of the pipe and fluid are neglected. Planar motion is considered. The pipe is inclined with horizontal axis by angle $\theta$. The specification and numerical value of parameters of the pipe and fluid under consideration in this study are listed in Table 1. 


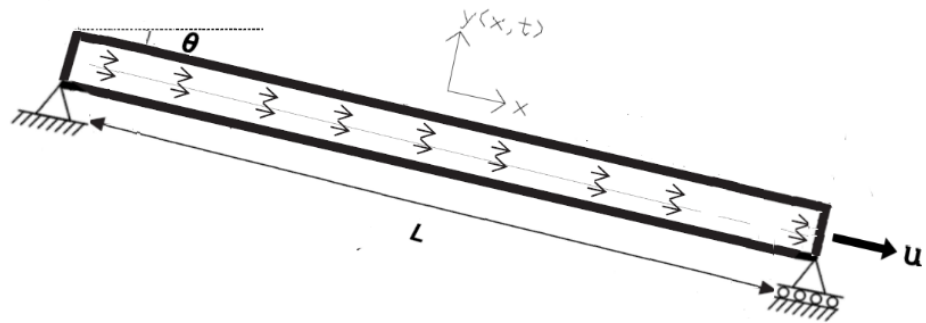

Fig. 1. Schematic of pipe with simply support

\begin{tabular}{|c|c|}
\hline Specification & Value \\
\hline Material & Polypropylene-Random (PP-R) \\
\hline Fluid & Water \\
\hline Inner diameter $\mathrm{D}_{\mathrm{i}}(\mathrm{m})$ & 0.018 \\
\hline Thickness t (m) & 0.0035 \\
\hline Length L (m) & $0.5-1.25$ \\
\hline Aspect ratio (length to outer dimeter) $\mathrm{L} / \mathrm{D}_{\mathrm{o}}$ & $\begin{array}{l}(20-50) \mathrm{D}_{\mathrm{o}} \\
0.8 \text { at } 25^{\circ} \mathrm{C}\end{array}$ \\
\hline Modulus of elasticity E (GPa) & $\begin{array}{l}0.38 \text { at } 50^{\circ} \mathrm{C} \\
0.23 \text { at } 70^{\circ} \mathrm{C}\end{array}$ \\
\hline Density of pipe $\rho_{p}\left(\mathrm{Kg} / \mathrm{m}^{3}\right)$ & 909 \\
\hline Density of fluid $\rho_{\mathrm{f}}\left(\mathrm{Kg} / \mathrm{m}^{3}\right)$ & 1000 \\
\hline Coefficient of expansion $\alpha(1 / \mathrm{K})$ & $0.3 \times 10^{-4}$ \\
\hline
\end{tabular}

$E I \frac{\partial^{4} y}{\partial x^{4}}-\left[(m+M)(L-x) g \sin \theta-F_{n}-m u^{2}\right] \frac{\partial^{2} y}{\partial x^{2}}+(m+M) g \sin \theta \frac{\partial y}{\partial x}+(m+M) g \cos \theta+2 m u \frac{\partial^{2} y}{\partial x \partial t}+(m+M) \frac{\partial^{2} y}{\partial^{2} t}=0$

To deduce the equation of motion of the inclined, heated pipe, the following assumptions were considered:

- The transverse motion (y-axis) of the pipe was considered only.

- The fluid is assumed to be incompressible, non-viscous, and Newtonian fluid.

- The mean velocity of the flowing fluid along the pipe is constant.

- A linear relationship between the strains and displacements was adopted.

- The material of the pipe is assumed to be isotropic and the internal damping is negligible.

- The cross-section dimensions of the pipe are assumed to be constant.

- The friction between the flow and the pipe wall is negligible.

The full derivation of the fourth order partial differential equation of motion governing the dynamics of a pipe containing fluid segment with the boundary conditions can be found in ref. (Mohmmed et al., 2021).

Where $\mathrm{g}$ is the gravitational constant, $\mathrm{E}$ is the modulus of elasticity, I is the moment of inertia, $y(x, t)$ is the transverse displacement of the pipe; $x$ and $t$ are the axial coordinate and time, respectively, $F_{n}$ is thermal loading due to temperature variation and can be determined by Equation (2) (Pytel, and Singer, 1987):

$F_{n}=\alpha E A_{o} \Delta T$

Where $\alpha$ : is coefficient of thermal expansion $\left(1 /{ }^{\circ} \mathrm{C}\right)$, $\Delta \mathrm{T}=\mathrm{T}_{\mathrm{x}}-\mathrm{T}_{\mathrm{i}}$ : is the temperature change $\left({ }^{\circ} \mathrm{C}\right) . \mathrm{T}_{\mathrm{i}}$ : is the room temperature $\left({ }^{\circ} \mathrm{C}\right) .: \mathrm{T}_{\mathrm{x}}$ : is the instantaneous temperature of the pipe $\left({ }^{\circ} \mathrm{C}\right)$.

Equation (1) is consisting of eight terms, which stand for the following: the pipe stiffness, the gravity force, the thermal force (which emerges as a result of temperature variation in the pipe), the centrifugal force (which appears because of fluid acceleration through the deformed pipe), the fifth and sixth terms represent the static force due to the gravity effect, the Coriolis force, and the pipe inertia.

Usually in literature of fluid-structure interaction mechanics, where fluid induced vibrations are investigated, Equation (1) can be rewritten in non-dimensional form as Equation (3).

$$
\begin{aligned}
& \frac{\partial^{4} \eta}{\partial \xi^{4}}-\left[(1-\xi) \bar{G} \sin \theta-\bar{F}_{n}-U^{2}\right] \frac{\partial^{2} \eta}{\partial \xi^{2}}+ \\
& \bar{G} \sin \theta \frac{\partial \eta}{\partial \xi}+\bar{G} \cos \theta+2 \beta^{1 / 2} U \frac{\partial^{2} \eta}{\partial \xi \partial \tau}+\frac{\partial^{2} \eta}{\partial^{2} \tau}=0
\end{aligned}
$$


Or $\eta^{\prime \prime \prime \prime}-\left[(1-\xi) \bar{G} \sin \theta-\overline{F_{n}}-U^{2}\right] \eta^{\prime \prime}+\bar{G} \sin \theta \eta^{\prime}+2 \beta^{1 / 2} U / \&+\bar{G} \cos \theta$

$\eta=\frac{y}{L}, \quad \xi=\frac{x}{L}, \beta=\frac{m}{m+M}, \bar{G}=\frac{(m+M) g L^{3}}{E I}$,

$U=\left(\frac{m}{E I}\right)^{\frac{1}{2}} u L, \tau=\frac{1}{L^{2}}\left(\frac{E I}{m+M}\right)^{\frac{1}{2}}, \overline{F_{n}}=\frac{F_{n} L^{2}}{E I}$

$(\eta, \xi)$ are non-dimensional position in transverse and longitudinal direction respectively, $\beta$ is mass ratio, $\bar{G}$ is non-dimensional gravity effect, $U$ is non-dimensional flow velocity, $\tau$ is non-dimensional time, $\overline{F_{n}}$ is non-dimensional thermal loading.

Herein, the prime ( )' indicates a derivative with respect to $\xi, \partial() / \partial \xi$, and the $\operatorname{dot}(\mathrm{g})$ indicates a derivative with respect to $\tau, \partial() / \partial \tau$.

\subsection{Analytic Solution}

Analytic solution of Equation (4) using mixed finite Fourier sine and Laplace transforms methods described in this section. Equation (4) was discretised by using the separation of variables technique, in order to transform it to ordinary differential equations. The term $\eta(\xi, \tau)$ is decomposed into space and time as follows.

$\eta(\xi, \tau)=Y(\xi) q(\tau)$

Where $q(\tau)$ is the generalized coordinate of the system and $Y(\xi)$ represents a trial/comparison function that satisfy both the geometric and natural boundary conditions. By substituting Equation (6) in Equation (4), and conducting the finite Fourier sine transform and Laplace transform assuming $q(0)$, and $\&(0)$ equal to zero, yield;

$$
\int_{0}^{1}\left\{\begin{array}{l}
Y^{\prime \prime \prime}(\xi) q(s)-\left[\bar{G} \sin \theta-\overline{F_{n}}-U^{2}\right] Y^{\prime \prime}(\xi) \\
q(s)+\bar{G} \sin \theta \xi Y^{\prime \prime}(\xi) q(s)+\bar{G} \sin \theta Y^{\prime}(\xi) \\
q(s)+\frac{\bar{G}}{s} \cos \theta+2 \beta^{1 / 2} U Y^{\prime}(\xi) s q(s)+ \\
Y(\xi) s^{2} q(s)
\end{array}\right\}=0
$$

Where $s$ indicates a complex number in the Laplace domain, $Y(\xi)$ is varying according to boundary condition, for simply support case:

$Y(\xi)=\sqrt{2} \sin \left(\lambda_{r} \xi\right), \sqrt{2} \sin \left(\lambda_{r} \xi\right)=0 \Rightarrow \lambda_{r}=\frac{n \pi}{L}$

The boundary condition for simply support are

$Y^{\prime \prime}(\xi)=Y(\xi)=0$ at $\xi=1$ and $\xi=0$

To simplify finding the integral transformation for each term in Equation (5), a polynomial function can be applied for this type of support system:

$Y(\xi)=a_{0}+a_{1} \xi+a_{2} \xi^{2}+a_{3} \xi^{3}+a_{4} \xi^{4}$
Where, $a_{0}, a_{1}, a_{2}, a_{3}$, and $a_{4}$ are constants to be determined by applying boundary conditions. Then, by applying the boundary conditions and using orthogonal rule, for $a=1$, we obtained $a_{4}=4.5$ for the first mode. By performing integration and rearrange Equation (7), gives;

$$
\left[\begin{array}{l}
s^{2}\left(\frac{24}{n^{5} \pi^{5}}\left(1+(-1)^{n+1}\right)\right)+ \\
\left(\begin{array}{l}
s\left(\begin{array}{l}
2 \beta^{1 / 2} U \\
\frac{1}{n \pi}\left(1+(-1)^{n}\right) \\
+\frac{12}{n^{3} \pi^{3}}\left(1+(-1)^{n}\right)
\end{array}\right) \\
+\frac{24}{n \pi}\left(1+(-1)^{n+1}\right)-\left(\overline{F_{n}}+U^{2}\right) \\
\left(\begin{array}{l}
\frac{24}{n^{3} \pi^{3}} \\
\left(\begin{array}{l}
\left.1+(-1)^{n+1}\right) \\
+
\end{array}\right) \\
\left(\begin{array}{l}
\frac{24}{n^{3} \pi^{3}}\left(1+2(-1)^{n}\right)+\frac{1}{n \pi} \\
\left(1+(-1)^{n}\right)+\frac{12}{n^{3} \pi^{3}}\left(1+(-1)^{n}\right)
\end{array}\right.
\end{array}\right]
\end{array}\right]
\end{array}\right]
$$

Herein, $n$ denotes the mode number.

Let

$$
\begin{aligned}
& Z_{p 1}=2 \beta^{1 / 2} U\left(\frac{1}{n \pi}\left(1+(-1)^{n}\right)+\frac{12}{n^{3} \pi^{3}}\left(1+(-1)^{n}\right)\right) \\
& Z_{p 2}=\frac{24}{n \pi}\left(1+(-1)^{n+1}\right)-\left(\overline{F_{n}}+U^{2}\right)\left(\begin{array}{l}
\frac{24}{n^{3} \pi^{3}} \\
\left(1+(-1)^{n+1}\right)
\end{array}\right) \\
& +\bar{G} \sin \theta\left(\begin{array}{l}
\frac{24}{n^{3} \pi^{3}}\left(1+2(-1)^{n}\right)+\frac{1}{n \pi} \\
\left(1+(-1)^{n}\right)+\frac{12}{n^{3} \pi^{3}}\left(1+(-1)^{n}\right) \\
+\frac{24}{n^{3} \pi^{3}}\left(1+(-1)^{n+1}\right)
\end{array}\right)
\end{aligned}
$$


$\therefore\left[s^{2}\left(\frac{24}{n^{5} \pi^{5}}\left(1+(-1)^{n+1}\right)\right)+s Z_{p 1}+Z_{p 2}\right] q(s)$
$=-\frac{1}{a_{4}} \frac{\bar{G}}{s} \cos \theta \cdot 1(f)$

Moreover,

$$
\begin{aligned}
& \text { Let } \phi_{p 1}=\frac{Z_{p 1}}{\frac{24}{n^{5} \pi^{5}}\left(1+(-1)^{n+1}\right)} \\
& \phi_{p}^{2}=\frac{Z_{p 2}}{\frac{24}{n^{5} \pi^{5}}\left(1+(-1)^{n+1}\right)}
\end{aligned}
$$$$
\therefore\left[s^{2}+s \phi_{p 1}+\phi_{p}^{2}\right] q(s)=\frac{-\frac{1}{a_{4}} \frac{\bar{G}}{s} \cos \theta .1(f)}{\frac{24}{n^{5} \pi^{5}}\left(1+(-1)^{n+1}\right)}
$$$$
\Rightarrow q(s)=\frac{\frac{-\frac{1}{a_{4}} \frac{\bar{G}}{s} \cos \theta \cdot 1(f)}{\frac{24}{n^{5} \pi^{5}}\left(1+(-1)^{n+1}\right)}}{\left[s^{2}+s \phi_{p 1}+\phi_{p}^{2}\right]}
$$

By applying the finite Fourier sine and Laplace inversion, the dynamic response $\eta(\xi, \tau)$ can be expressed as

$$
\eta(\xi, \tau)=Y(\xi) \frac{-\bar{G} \cos \theta \cdot \mathrm{F}_{p}(\tau)}{\frac{24}{n^{4} \pi^{4}} a_{4}}
$$

Where,

$$
\begin{aligned}
& \mathrm{F}_{p}(\tau)=\left(\frac{1}{\alpha_{p 1} \alpha_{p 2}}+\frac{1}{\alpha_{p 1} \alpha_{p 2}\left(\alpha_{p 2}-\alpha_{p 1}\right)}\left(\alpha_{p 1} e^{-\alpha_{p 2} \tau}-\alpha_{p 2} e^{-\alpha_{p 1} \tau}\right)\right) \\
& \alpha_{p 1}=\frac{\phi_{p 1}}{2}+i \sqrt{\phi_{p}^{2}-\frac{\phi_{p 1}^{2}}{4}} \\
& \alpha_{p 2}=\frac{\phi_{p 1}}{2}-i \sqrt{\phi_{p}^{2}-\frac{\phi_{p 1}^{2}}{4}}
\end{aligned}
$$

\subsection{MATLAB Algorithm and Flow Chart}

To calculate the dynamic response represented by Equation (16), the MATLAB 2019b software was used. The flow chart presented in Fig. 2 show coding program based on following algorithm steps:

- Define the constants $g, \rho_{\mathrm{f}}, \rho_{\mathrm{p}}, \mathrm{D}_{\mathrm{i}}, \mathrm{D}_{\mathrm{o}}, \mathrm{T}_{\mathrm{i}}, \alpha$ by using Table 1.

- Calculate, A, A $, \mathrm{m}, \mathrm{M}$, I by using the following formulas:

$$
\begin{aligned}
& A=\frac{\pi}{4} D_{i}^{2}, \quad A_{p}=\frac{\pi}{4}\left(D_{o}^{2}-D_{i}^{2}\right), \quad m=\rho_{f} A, \\
& M=\rho_{p} A_{p}, I=\frac{\pi}{64}\left(D_{o}^{4}-D_{i}^{4}\right)
\end{aligned}
$$

- Define the variables $\mathrm{u}, \mathrm{L} / \mathrm{D}_{\mathrm{o}}, \theta, \mathrm{Tx}$, and $\mathrm{t}$.

- Define E (set value of E from Table 1 according to temperature $\left.\mathrm{T}_{\mathrm{x}}\right), \mathrm{n}(\operatorname{set} \mathrm{n}=1)$, and $a_{4}\left(\operatorname{set} a_{4}=1\right)$.

- Calculate $F_{n}$ by using the Equation (2).

- Calculate the dimensionless parameters using the formulas in Equation (5).

- Set the interval of the dimensionless position $\xi$ between (0-1) with step 0.01 .

- Find $\mathrm{Z}_{\mathrm{p} 1}$ and $\mathrm{Z}_{\mathrm{p} 2}$ using the Equation (12) (a and b).

- Find $\phi_{p 1}$ and $\phi_{p}^{2}$ using the Equation (14) (a and b).

- Calculate the value of the terms, $a_{p 1}, a_{p 2}$, and $\mathrm{F}_{p}(\tau)$ using the Equations (18) (a and b), and (17).

- Find the dynamic response $\eta(\xi, \tau)$ of the system from the relation in Equation (16).

- Plot the results.

\section{RESULTS AND DISCUSSION}

In many studies to date about the stability conditions and the general vibration behavior that associate with unstable fluctuation of pipe-fluid systems, investigators have been preoccupied with two fundamental issues; building up the general manner of the dynamic deflection of the system; establishing the region where such a solution holds validity.

In accordance with the linear theory, the latter issue includes finding the critical value of the internal fluid velocity for the pipe-fluid system, whereas the former one has unraveled curious and sudden paradoxical manners of flow. The difficulties that may be faced mostly associate with the methodology employed in treating with these problems wherein dependence was put on tackling abridged equations whose solutions were supported with powerful and masterly explanation of physical and numerical practical data.

In current work some of the well-known results (Equation (7)) were recovered from a straightforward application of mixed Fourier-Laplace transforms to the full linear equations. This give us a chance to:

- get accurate form and explicit representations for the well-known variables of flow like the system's dynamic deflection.

- Determining the dynamic response in a relatively easier method, which make us to avoid the tedium of laborious numerical work.

- A comparative parametric analysis of the variables included in the solution can be simply performed with avoid heavy dependence on numerical results,

- insurance, from the beginning., that the derived solution corresponds with the complete configuration and profile of the whole pipe length hence the 


\section{International Journal of Applied Science and Engineering}

Mohmmed et al., International Journal of Applied Science and Engineering, 18(6), 2021133

development of the final configuration as a direct result of the initial pipe profile does not constitute an issue.

In this part, the results obtained from the analytic solution of the governing equation of an inclined, heated, pinned ends pipe conveying fluid, Equation (1), are presented. Especially, the effects of support angle, aspect ratio, and temperature on the natural frequencies and dynamic deflection are emphasized. The results simulated in MATLAB 2019b software. Results are presented for a pipe manufactured from polypropylene random-copolymer (PPR) material, and the fluid used in all cases was water.

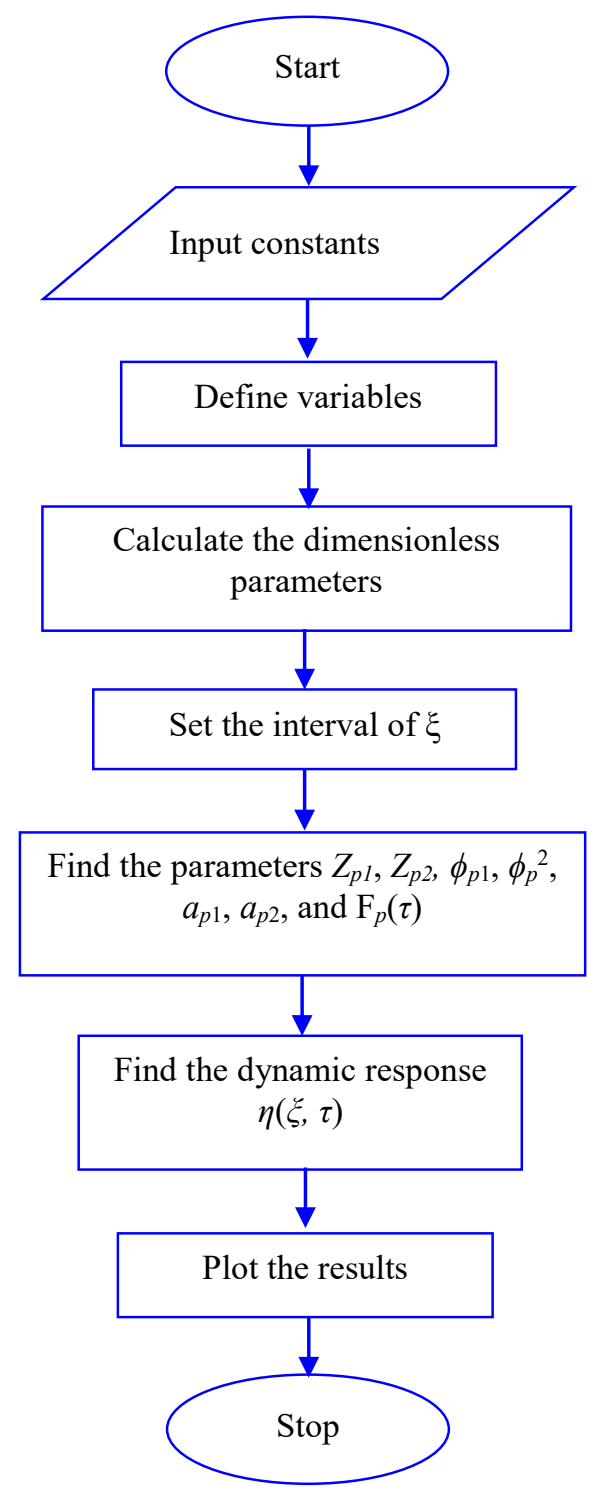

Fig. 2. Flow chart of the MATLAB code

Table 2. Comparison of Max. dimensionless dynamic deflection of simply supported pipe with different fluid velocities at $\xi=0.5$

\begin{tabular}{ccc}
\hline $\begin{array}{c}\text { Non-dimensional Fluid } \\
\text { velocity U }\end{array}$ & $\begin{array}{c}\text { Hybrid finite Fourier sine-Laplace } \\
\text { transform solution (exact) }\end{array}$ & $\begin{array}{c}\text { Galerkin's method solution with } \mathrm{k}=2 \\
\text { (approximate) }\end{array}$ \\
1.5 & $1.78082 \times 10^{-7}$ & $1.86982 \times 10^{-7}$ \\
2 & 0.00117949 & 0.00161896 \\
2.5 & 0.00367356 & 0.00318154 \\
3 & 0.00026963 & 0.00035296 \\
\end{tabular}




\subsection{Comparing the Proposed Analytic Solution with that of Galerkin}

To verify the correctness of the integral transforms technique (ITT), the solution obtained by combining of Laplace and finite Fourier sine transforms was compared with that of Galerkin method. A straight, pinned-pinned pipe containing flowing fluid with inclination angle $(\theta=0)$ and thermal loading $\left(F_{n}=0\right)$ was adopted in this comparison. The dynamic deflection of the simply supported pipe was calculated in current study by the formula shown in Equation (16). The following non-dimensional parameters are used; $\beta=0.54, \mathrm{~L}=1, \tau=2$.

To calculate the dynamic deflection by Galerkin method, suppose the solution of Equation (1) can be expressed as: $\eta(\zeta, \tau)$ can be approximated by:

$\Pi(\xi, \tau)=\sum_{i=1}^{k} \Gamma_{i}(\xi) \Lambda_{i}(\tau)$

Where $\Gamma_{i}(\xi)$ represent the Eigen functions of simply supported beam, and $\Lambda_{i}(\tau)$ is the i-th generalized coordinate of the system. $k$ represents the number of terms used in the Galerkin expansion. By substituting Equation (19) in Equation (3), and conducting the orthogonal rule, the $4^{\text {th }}$ order partial differential equation of pipe containing flowing fluid was decreased to an ordinary differential equation. The ordinary differential equations were solved by Newmark and Newton-Raphson iteration method. More details of the Galerkin method can be found in ref. (Hill and Swanson, 1970). Table 2 presents the results of both methods and shows good convergence between them. The advantage of using a combination of Fourier and Laplace transforms is that it provides a more accurate solution (exact solution).

\subsection{Dynamic Response}

Lateral bending or buckling take places even in the absence of flow for a simply supported pipe. This is because the effects of the weight of the pipe and the reactions at its two support ends which lead to the deformation of the neutral axis of the pipe. Therefore, for a pipe sealed at both ends and contained an entrained static fluid, the lateral bending degree rises when the weight of the entrained fluid increases. However, the bending orientation can be altered with changing the fluid velocity values if the dynamic buckling took place when the fluid is flowing inside the pipe. For the case of the fundamental buckling mode $n=1$, Fig. 3 , presents the system's dynamic responses against the dimensionless position for various internal flow velocity and at constant aspect ratio $\mathrm{L} / \mathrm{D}=20$. It is clear from figure, the curves, that describe the physics of dynamically excited pinned-pinned pipe-fluid system, are parabolas and as the pipe is dynamically moving as result of the fluid flow, on basis of its magnitude, the characteristics of the pipe alternate to obtain convex and zero (straight line) curvatures. Also, it can be seen from figure that the maximum transverse deformation occurs at the midpoint of the pipe span $(\xi \approx 0.5)$ at any internal flow velocity, and this transverse deformation become higher with increasing internal fluid velocity. This is because the induced follower compressive force. However, when the internal fluid velocity convergences of its critical value, the transverse deformation decrease to zero and then suddenly jump to highest deflection amplitude, that refers to that the pipe is going to lose stability.

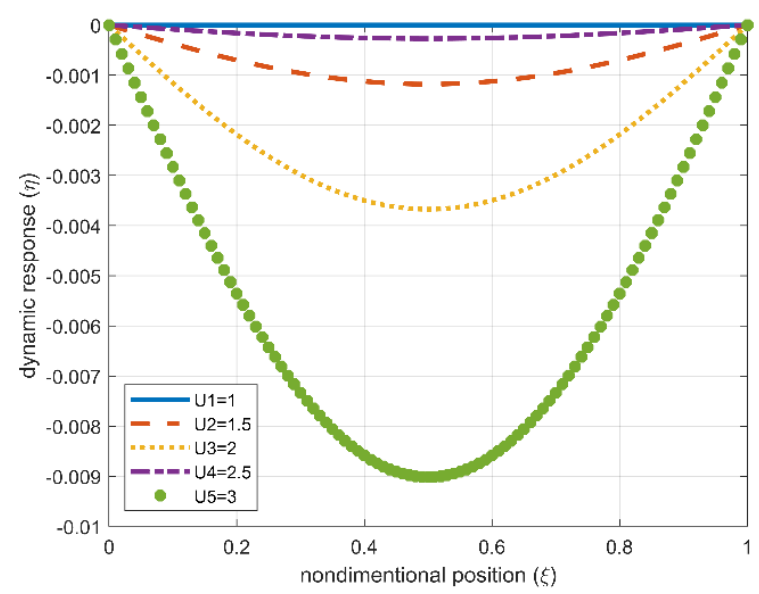

Fig. 3. Dynamic response versus dimensionless position $\xi$, with various dimensionless internal flow velocity $\mathrm{U}$, at conditions: $\mathrm{n}=1, \mathrm{~L} / \mathrm{D}=20, \tau=2, \mathrm{~T}=25^{\circ} \mathrm{C}, \theta=0^{\circ}$

Then, in Fig. 4, the dynamic response versus the aspect ratios with various internal flow velocity is evaluated. The dynamic response increases as aspect ratios increases. With the aspect ratios increasing, the deflection is increasing faster with higher internal flow velocity. This means that at a higher aspect ratio, a pipe - fluid system will be reaching the divergence instability state more fastly with increasing the internal flow velocity.

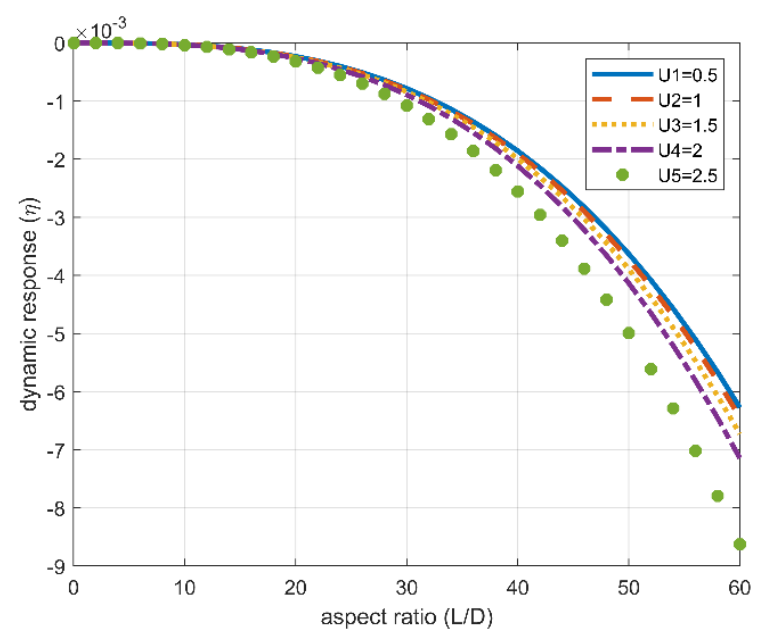

Fig. 4. Dynamic response $\eta$ versus aspect ratio $L / D$, with various dimensionless internal flow velocity $\mathrm{U}$, at conditions: $\mathrm{n}=1, \tau=0.2, \mathrm{~T}=25^{\circ} \mathrm{C}, \theta=0^{\circ}$

Fig. 5 shows the effect of the different support angles on the dynamic response at uniform fluid flow velocity $U=1$ 


\section{International Journal of Applied Science and Engineering}

Mohmmed et al., International Journal of Applied Science and Engineering, 18(6), 2021133

and specific aspect ratio $\mathrm{L} / \mathrm{D}=20$. Figure exhibits a convex or concave parabolic curvature depending on the value of angle below or above $90^{\circ}$. When the angle below $90^{\circ}$, the vibration deflection reduces with increase the inclined angle and verse versa above the $90^{\circ}$. This can be explained as following, the gravitational load resulting from the weight of pipe segment and fluid for inclined pipe is calculated by its horizontal and vertical components along the pipe length. The effect of the vertical (transverse) component, which produces the transverse deformation of the pipe, can be reduced by the horizontal (axial) component. Hence, the deflection decrease with rising angle inclination due to decrease magnitude of the transverse weight component of fluid and pipe segment.

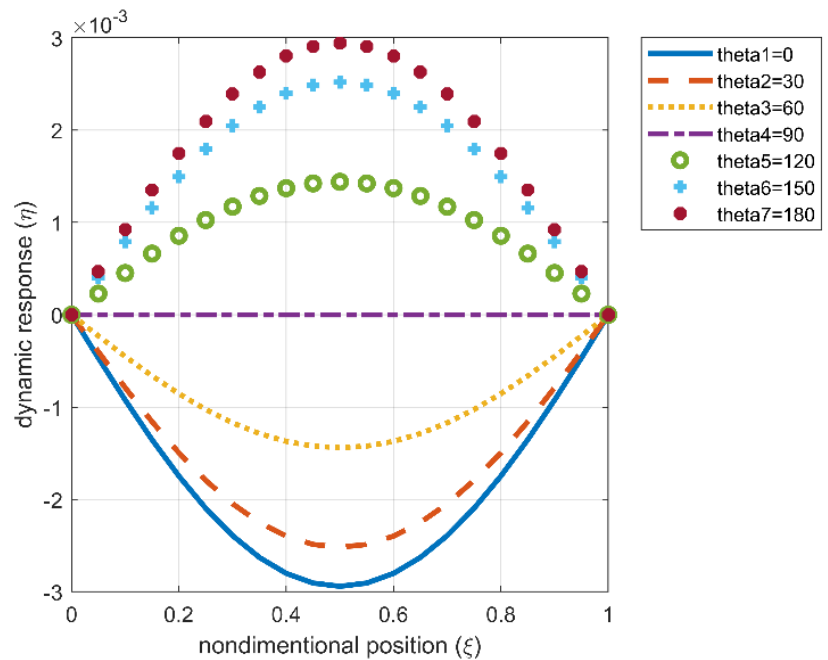

Fig. 5. The variation of the dynamic response $\eta$ with pipe length for different support angle $\theta$ for the case: $n=1, L / D$

$$
=20, \tau=0.2, \mathrm{U}=1
$$

Naturally, it is expected that the variation in the temperature can be having some influence on the transverse displacement of the pipe-fluid systems. In this respect, Fig. 6 illustrates the response of the dynamic deflection to the dimensionless length of the pipe under different temperature. Particularly, the increasing in the temperature has been resulted in the rising in the transverse displacement with the maximum deflection being observed at the midpoint of the pipe span $(x=0.5 \mathrm{~L})$. This is attributed to the combined influence of thermally induced compressive stress and the similar stress causing by the internal fluid velocity. At the same time, the level of dynamic deflection for the same temperature variation reduces with increase in inclination angle as illustrated in Fig 7.

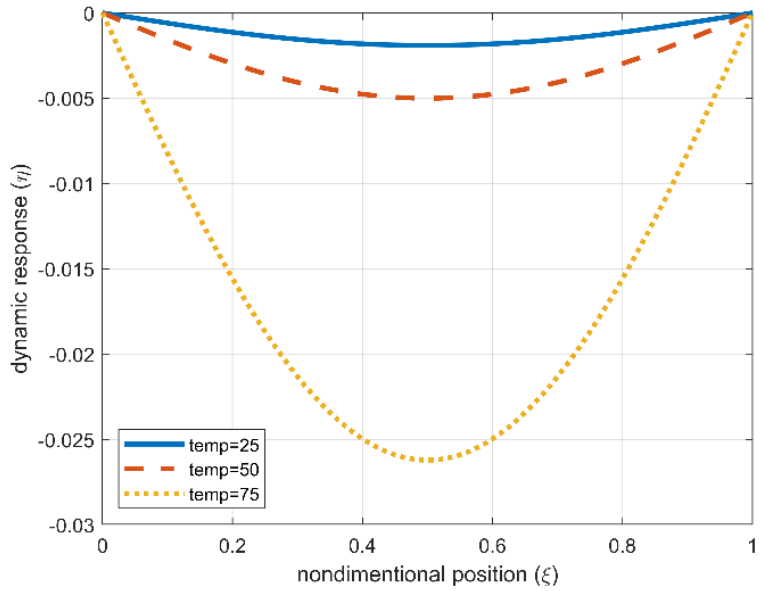

Fig. 6. The variation of the dynamic response $\eta$ with pipe length for different temperature for the case: $\mathrm{n}=1, \theta=0^{\circ}$, $\mathrm{L} / \mathrm{D}=20, \tau=0.2, \mathrm{U}=1$

On the other hand, it is noticed from Fig. 8 that when the aspect ratio is higher, the transverse displacement increases more dramatically with the increasing the temperature. With increasing the temperature, the maximum transverse displacement is increasing faster with larger aspect ratio $\mathrm{L} / \mathrm{D}$ values. This means that the stability of the pipe conveying fluid for a certain temperature, can be lose more easily with a higher aspect ratio.

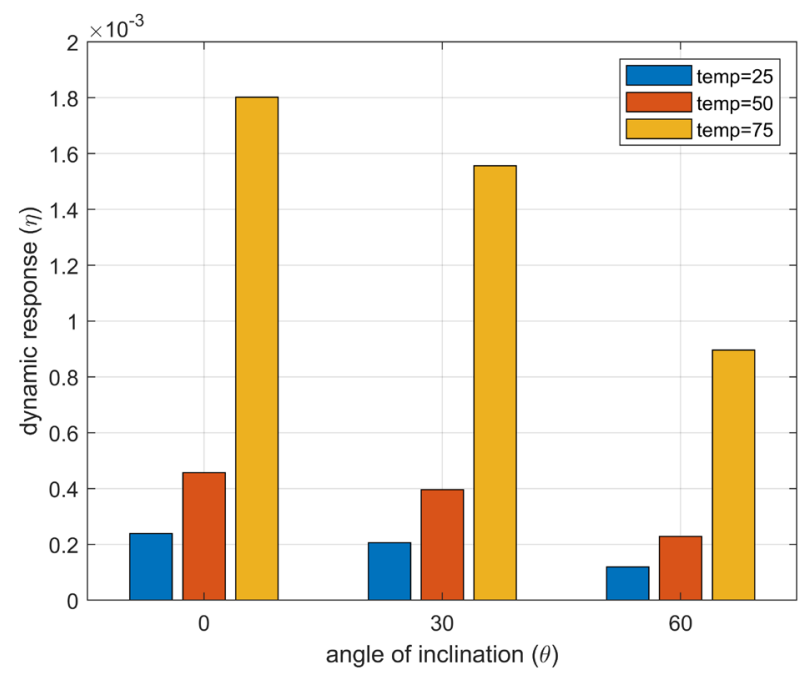

Fig. 7. The variation of the dynamic response $\eta$ with support angle $\theta$ for different temperature for the case: $\mathrm{n}=$

$$
1, \tau=0.2, \mathrm{~L} / \mathrm{D}=20, \mathrm{U}=1
$$


Mohmmed et al., International Journal of Applied Science and Engineering, 18(6), 2021133

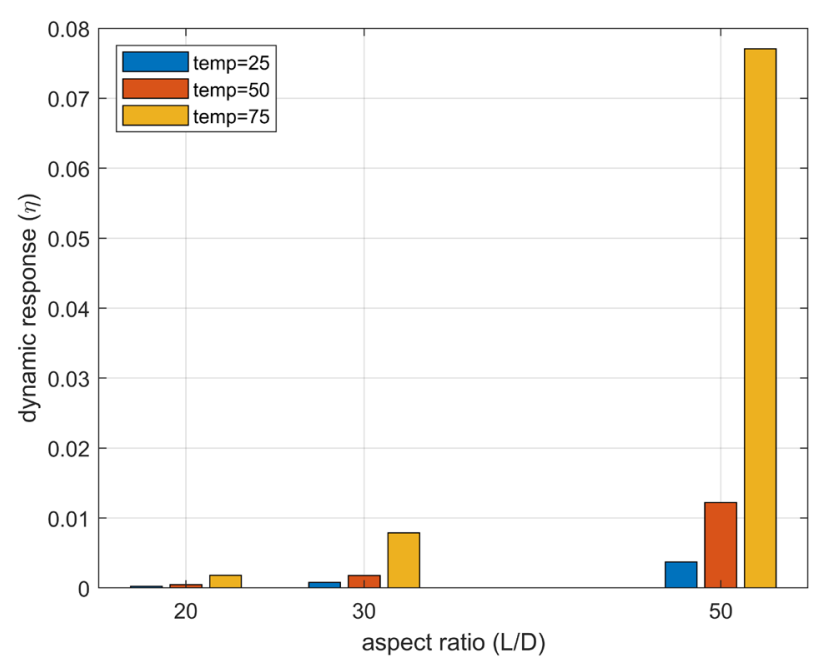

Fig. 8. The variation of the dynamic response $\eta$ with aspect ratio $L / D$ for different temperature for the case: $n=$ $1, \theta=0^{\circ}, \mathrm{U}=1, \tau=0.2$

Fig. 9 displays the dynamic response of the pipe containing flowing fluid to the dimensionless fluid velocity for different time. For this case other parameters such as the angle support of pipe, temperature of pipe and the pipe length are kept constant. It is observed that the general behaviour of dynamic response of pipe tends to increase with increasing the time and going to divergence (lose stability) when approach to the critical velocity. However, with more increasing time, the behaviour becomes more complex and the pipe undergoes oscillation and begins lose stability in its pre-buckled state. this behaviour can be associated with develop interchange of energy between the generated Coriolis force and the system, which leads to emerge symmetric and antisymmetric oscillations.

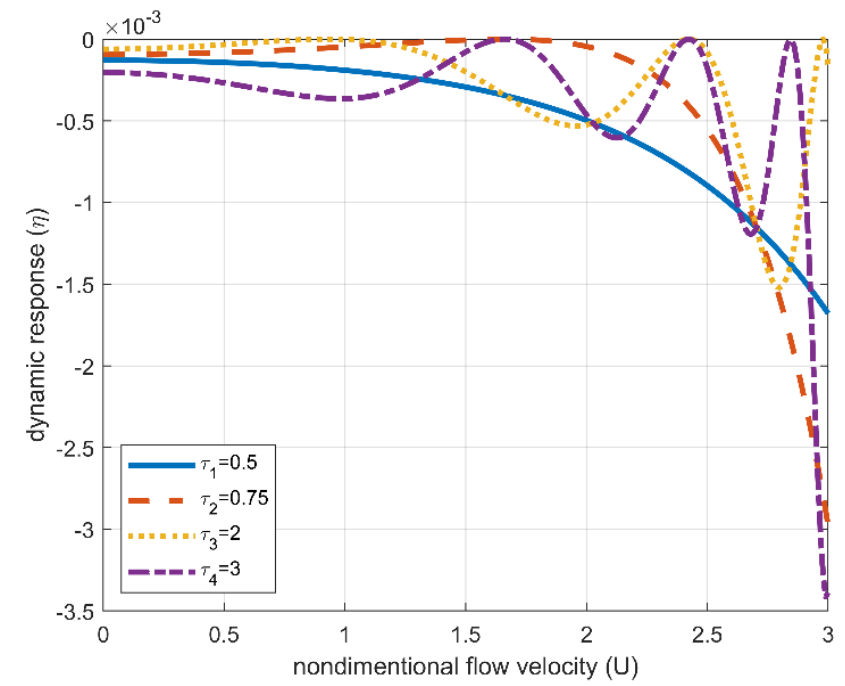

Fig. 9. The variation of the dynamic response $\eta$ with flow velocity $U$ for different dimensionless time $\tau$ for the case: $\mathrm{n}=1, \theta=0^{\circ}, \mathrm{L} / \mathrm{D}=20, \mathrm{~T}=25^{\circ} \mathrm{C}$

\section{CONCLUSION}

In current work, the problem of conveyance of fluid in straight pipes laid on the pinned support that inclined with the horizontal axis by various angles have been analyzed. Especially, dynamic deflection has been studied in relation to the modulating influences of internal fluid velocity, aspect ratio (length to external diameter of pipe), support angle, and thermal load. As the fluid velocity increases, the effects of pipe wall temperature variation, aspect ratio, and inclined angle, become important to designing engineers. In tackling this problem, a set of governing differential equations, which recognizes the fact that pumping of such fluid sets the pipe into transverse motions, have been derived. By making use of generalized integral transform approach (via mixed Fourier-Laplace transforms method), the coupled partial differential equations are solved and explicit closed form solutions of the inclined pipe conveying fluid system under thermal loading are obtained. By the linearized solution of these equations, it can be found the amount of dynamic deflection (transverse displacement) along the pipe. Through the theory and numerical simulations for present study about pipe conveying fluid, it can be drawn the following main conclusions:

- The mixed finite Fourier sine-Laplace transforms technique offers a powerful tool to obtain the dynamic characteristic of pipe conveying fluid.

- The fluid velocity can significantly affect the dynamic deflection (dynamic response) of the pipe.

- The vibration deflection (transverse displacement) is maximum at the midpoint of the pipe and increase with the increasing of internal flow velocity before the reaching their critical value.

- Increasing the pipe temperature leads to remarkable rise in vibration deflection.

- The maximum transverse displacement is larger at higher aspect ratio. At the same time, with continue increasing the fluid velocity, the maximum transverse displacement is increasing faster with higher aspect ratio.

- Increasing inclined angle with the horizontal axis from $0^{\circ}$ up to $90^{\circ}$ will lead to reduce the vibration deflection. This behavior is reversed at inclined angles larger than $90^{\circ}$ and below $180^{\circ}$.

\section{ACKNOWLEDGMENT}

We would like to thank Mechanical Engineering Dept. in University of Technology, Iraq to their support in this study.

\section{REFERENCES}

Ali, M., Alshalal, I., Mohmmed, J.H. 2021. Effect of the torsional vibration depending on the number of cylinders in reciprocating engines, International Journal of Dynamics and Control, 9, 901-909. 
Alshalal, I., Ali, M., Mohmmed, J.H., Rashed, A.A. 2021. Frequency response function curvature technique to detect damage for simply supported beam under harmonic excitation, AIP Conference Proceedings, 2386.

Ameen, K.A., Al-Dulaimi, M.J., Hatem, A.A. 2019. Experimental study of vibration on pipe conveying fluid at different end conditions for different fluid temperatures, Engineering and Technology Journal, 37, 512-515.

Amini, Y., Heshmati, M., Daneshmand, F. 2020. Dynamic behavior of conveying-fluid pipes with variable wall thickness through circumferential and axial directions, Marine Structures, 72.

Ashley, H., Haviland, G. 1950. Bending vibration of a pipe line containing flowing fluid, Journal of Applied Mechanics.

Askarian, A.R., Permoon, M.R., Shakouri, M. 2020. Vibration analysis of pipes conveying fluid resting on a fractional Kelvin-Voigt viscoelastic foundation with general boundary conditions, International Journal of Mechanical Sciences, 179.

Cao, J., Liu, Y., Liu, W. 2018. The effect of two cases of temperature distributions on vibration of fluid-conveying functionally graded thin-walled pipes, Journal of Strain Analysis.

ElNajjar, J., Daneshmand, F. 2020. Stability of horizontal and vertical pipes conveying fluid under the effects of additional point masses and springs, Ocean Engineering, 206.

Feodos'yev, P., 1951. Vibrations and stability of a pipe when a liquid flows through it, Znzhenernyi Sbornik, 10, 169.

Hill, J.L., Swanson, C.P. 1970. Effects of lumped masses on the stability of fluid conveying tubes, Journal of Applied Mechanics, 37, 494-497.

Housner, G.W. 1952. Bending vibration of a pipe line containing flowing fluid, Transactions of the ASME Journal of Applied Mechanics, 19, 205-208.

Huang, Q., Lin, T., Safarpour, M. 2020. Flow-induced vibration attenuation of a viscoelastic pipe conveying fluid under sinusoidal flow using a nonlinear absorber, Mechanics Based Design of Structures and Machines.

Jiang, T., Dai, H., Wang, L. 2020. Three-dimensional dynamics of fluid-conveying pipe simultaneously subjected to external axial flow, Ocean Engineering, 217.

Jweeg, M.J., Mohammad, Z.I. 2010. Vibration characteristics of different cross-section pipes with different end conditions, Engineering and Technology Journal, 28, 1634-1654.

Jweeg, M.J., Ntayeesh, T.J. 2016. Determination of critical buckling velocities of pipes conveying fluid rested on different supports conditions, International Journal of Computer Applications, 134, 34-42.

Kutin, J., Bajsić, I. 2014. Fluid-dynamic loading of pipes conveying fluid with a laminar mean-flow velocity profile, Journal of Fluids and Structures, 50, 171-183.
Li, B., Wang, Z., Jing, L. 2018, Dynamic response of pipe conveying fluid with lateral moving supports, Shock and Vibration, 2018, 1-17.

Li, B., Wang, Z., Jing, L. 2018. Dynamic response of pipe conveying fluid with lateral moving supports, Shock and Vibration.

Liang, F., Gao, A., Yang, X.D. 2020. Dynamical analysis of spinning functionally graded pipes conveying fluid with multiple spans, Applied Mathematical Modelling 83, 454-469.

Lu, Z.Q., Zhang, K.K., Ding, H., Chen, L.Q. 2020. Internal resonance and stress distribution of pipes conveying fluid in supercritical regime, International Journal of Mechanical Sciences 186.

Marakala, N., Appu Kuttan, K.K., Kadoli R. 2009. Experimental and theoretical investigation of combined effect of fluid and thermal induced vibration on vertical thin slender tube, Journal of Mechanical and Civil Engineering, 7, 63-68.

Mohmmed, J.H., Tawfik, M.A., Atiyah Q.A. 2021. Natural frequency and critical velocities of heated inclined pinned PP-R pipe conveying fluid, Journal of Achievements in Materials and Manufacturing Engineering, 107, 15-27.

Nejadi, M.M., Mohammadimehr, M., Mehrabi, M. 2021. Free vibration and stability analysis of sandwich pipe by considering porosity and graphene platelet effects on conveying fluid flow, Alexandria Engineering Journal, 60, 1945-1954.

Oke, W.A., Khulief, Y.A., 2020. Dynamic response analysis of composite pipes conveying fluid in the presence of internal wall thinning, Journal of Engineering Mechanics, 146, 1-11.

Paidousiss, M.P. 2008. The canonical problem of the fluidconveying pipe and radiation of the knowledge gained to other dynamics problems across applied mechanics, J. Sound Vib., 310, 462-492.

Païdoussis, M.P. 2014. Fluid structure interactions: Slender structures and axial flow, 1. Academic Press, London.

Tawfik, M.A., Kadhim, Z.K., Hammoudi, R.Y. 2009. Vibration analysis of sudden enlargement pipe conveying fluid with presence of heat flux, Engineering and Technology Journal, 27, 533-557.

Thomson, W.T. 1988. Theory of vibration with applications, Unwin Hyman Ltd, London.

Wang, Y., Wang, L., Ni, Q., Dai, H., Yan, H., Luo, Y. 2018. Non-planar responses of cantilevered pipes conveying fluid with intermediate motion constraints, Nonlinear Dynamics, 93, 505-524.

Zhai, H., Wu, Z., Liu, Yue, Y., Z. 2013. In-plane dynamic response analysis of curved pipe conveying fluid subjected to random excitation, Nuclear Engineering and Design, 256, 214-226. 